\title{
Evanescent-wave photoacoustic spectroscopy with optical micro/nano fibers
}

\author{
Yingchun Cao ${ }^{1}$, Wei Jin ${ }^{1, *}$, Lut Hoi Ho ${ }^{1}$, and Zhibo Liu ${ }^{1,2}$ \\ ${ }^{1}$ Department of Electrical Engineering, The Hong Kong Polytechnic University, Hong Kong, China \\ ${ }^{2}$ Key Laboratory of Weak Light Nonlinear Photonics, Ministry of Education, Teda Applied Physics School, \\ Nankai University, Tianjin 300457, China \\ *Corresponding author: eewjin@polyu.edu.hk
}

Received September 30, 2011; accepted November 22, 2011;

posted November 30, 2011 (Doc. ID 155538); published January 12, 2012

We demonstrate gas detection based on evanescent-wave photoacoustic (PA) spectroscopy with tapered optical fibers. Evanescent-field instead of open-path absorption is exploited for PA generation, and a quartz tuning fork is used for PA detection. A tapered optical fiber with a diameter down to the wavelength scale demonstrates detection sensitivity similar to an open-path system but with the advantages of easier optical alignment, smaller insertion loss, and multiplexing capability. (c) 2012 Optical Society of America

OCIS codes: $\quad 060.2370,300.6260,300.6380$.

Trace gas detection has drawn considerable attention recently because of its many important applications in environmental monitoring and industrial process control. Optical fiber sensors based on open-path absorption spectroscopy have been studied extensively and deployed for real-world applications [1]. The sensitivity of an open-path sensor improves with increasing the absorption path length. However, the improvement is limited because of difficulty associated with fabricating a long absorption cell. Evanescent-wave absorption spectroscopy using a D-shaped optical fiber has been studied but suffers from very low sensitivity as compared with open-path sensors [2]. Fiber tapers were also studied for evanescent-wave gas detection. However, the sensitivity is limited due to difficulty in fabricating a long fiber taper [3]. Microstructured optical fibers have the potential of achieving high sensitivity and possibly distributed gas detection along the fibers $[\underline{4}, \underline{5}]$, and active researches are ongoing in this area.

An alternative is photoacoustic spectroscopy (PAS), in which an acoustic pressure wave is generated by light absorption of gas molecules and detected by an acoustic transducer [6]. Among the variety of PAS systems, quartzenhanced (QE) PAS (QE-PAS) [7,8], which uses a resonant quartz tuning fork (QTF) as the acoustic transducer, has demonstrated outstanding performance. The photoacoustic (PA) signal may be further enhanced by use of a proper acoustic resonator [9-12].

However, in all of the PAS systems reported so far, open-path PA cells have been used and complex and precise collimating/focusing optics are needed to minimize the optical insertion loss and improve the efficiency of PA generation. In this Letter, we demonstrate evanescent-wave PAS (EPAS), which uses tapered optical micro/nano fibers (OMNFs) for PA generation. As the diameter of a fiber is tapered down to wavelength scale or smaller, a considerable portion of the optical mode power is located outside the OMNF [13], which can be exploited for PA generation. Because of their limited length, tapered OMNFs may not be efficient for highsensitivity gas detection based on absorption spectroscopy. However, they are well suited for PAS in which an acoustic transducer such as QTF converts localized
PA pressure into an electrical signal. The amplitude of the PA signal has no direct relationship with the absorption length. In an EPAS system, a tapered OMNF with a length of a few millimeters would be sufficient for highsensitivity gas detection if a small QTF is used. In the following, we demonstrate gas detection with QE-EPAS.

The fiber tapers were made by the flame-brushing technique from standard single-mode fibers (SMFs) $[14,15]$. By controlling the flame movement and fiber stretching rate, fiber tapers with diameters down to the subwavelength scale and low loss of $\sim 0.2 \mathrm{~dB}$ can be fabricated. An OMNF is threaded through the gap between the two prongs of a QTF, as shown in Fig. 1. Light is guided along the OMNF with a very small beam size, and no precise optical alignment is needed. Wavelengthmodulated light from a single longitudinal mode laser is transmitted to the OMNF, and the evanescent field is absorbed by the target gas, generating an acoustic pressure wave that is detected by the QTF.

The experimental setup is shown in Fig. 2. A distributed feedback (DFB) laser with a wavelength of $\sim 1532.8 \mathrm{~nm}$ was used as the light source. A ramp signal generated from a computer scans the laser wavelength across the absorption line periodically. Meanwhile, a sinusoidal signal modulates the laser wavelength at the frequency $f=f_{0} / 2$, where $f_{0}=32.75 \mathrm{kHz}$ is the resonant frequency of the QTF (KDS-DT38, $Q=6341$ at atmospheric pressure, $R=220 \mathrm{k} \Omega$ ) in our experient with dimensions of $6 \mathrm{~mm} \times 1.4 \mathrm{~mm} \times 0.2 \mathrm{~mm}$. The light signal is amplified by an erbium-doped fiber amplifier (EDFA) and split by a 10/90 fiber coupler. 10\% of the light is fed to the reference cell for calibrating gas concentration by

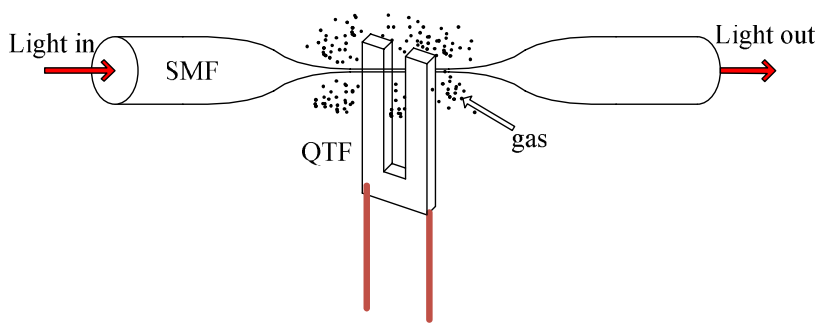

Fig. 1. (Color online) Schematic of QE-EPAS with an OMNF. 


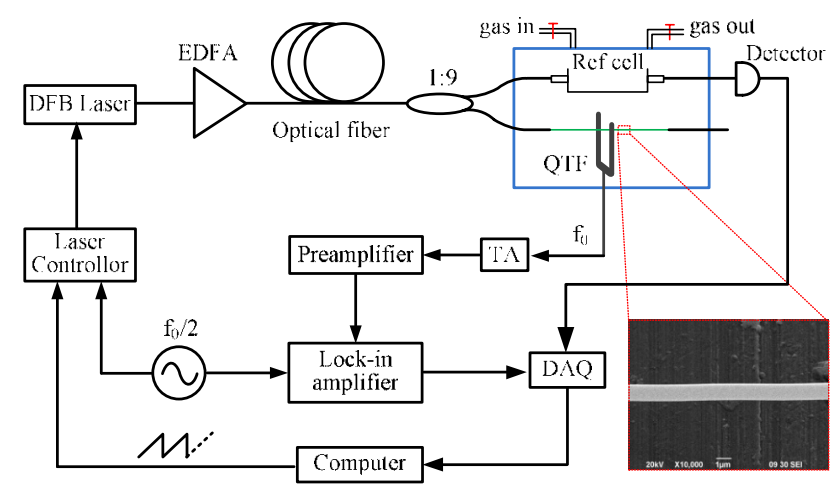

Fig. 2. (Color online) Experimental setup: EDFA, erbiumdoped fiber amplifier; QTF, quartz tuning fork; Ref cell, open-path cell with $2 \mathrm{~cm}$ absorption length; TA, transimpedance amplifier with a feedback resistor $R_{g}=10 \mathrm{M} \Omega$; DAQ, data acquisition device; $f_{0}$, resonant frequency of the QTF. Inset, scanning electron micrograph image of the OMNF.

direct absorption, while the remaining $90 \%$ to the OMNF for PA-based gas detection. For optimized PA detection, the OMNF was placed approximately in the middle of the gap between two prongs and $\sim 0.7 \mathrm{~mm}$ below the QTF opening [7]. The output from the QTF passes through a transimpedance amplifier (TA) with a feedback resistor of $10 \mathrm{M} \Omega$ and a preamplifier with a gain of 6 before entering into a lock-in amplifier for second-harmonic detection. The time constant of the lock-in amplifier was set to $30 \mathrm{~ms}$ with an $18 \mathrm{~dB} /$ Oct slope filter, corresponding to a detection bandwidth of $\Delta f=3.125 \mathrm{~Hz}$.

Three fiber tapers with different waist diameters were tested for $\mathrm{C}_{2} \mathrm{H}_{2}$ detection in air. The absorption line used is the $\mathrm{P}(13)$ line at $6523.88 \mathrm{~cm}^{-1}$ with a line intensity of $1.035 \times 10^{-20} \mathrm{~cm}^{-1} /\left(\mathrm{mol} \mathrm{cm}^{-2}\right)$. The measurement was performed at atmospheric pressure. Figure 3 shows the normalized second-harmonic signals for the three fiber tapers when the laser wavelength was scanned across the absorption line. The parameters of the three fiber tapers and the results of the QE-EPAS are listed in Table 1 . For the fiber taper with diameter of $1.1 \mu \mathrm{m}$, the measured (normalized) maximum second-harmonic output signal is $0.0317 \mathrm{mV} /(\mathrm{W}$ ppmv $)$. Considering the similar absorption line intensity of $\mathrm{H}_{2} \mathrm{O}$ at $7176.41 \mathrm{~cm}^{-1}$ and the gain factor of the preamplifier used in [12], this value may be regarded as comparable to the value of QE-PAS with a QTF alone without the use of an acoustic resonator. This suggests that the QE-EPAS would achieve a similar performance as an open-path PAS system.

The percentage of light power in the evanescent field as a function of the fiber diameter was calculated and is shown in Fig. $\underline{4}$ as a solid curve. The maximum secondharmonic outputs for the three tapers, which are normal-

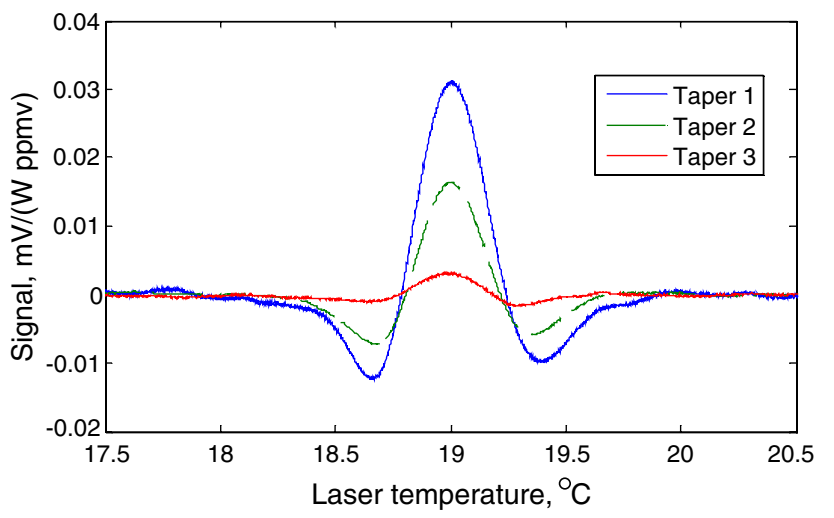

Fig. 3. (Color online) Normalized second-harmonic output signal for the QE-EPAS system with three different OMNFs.

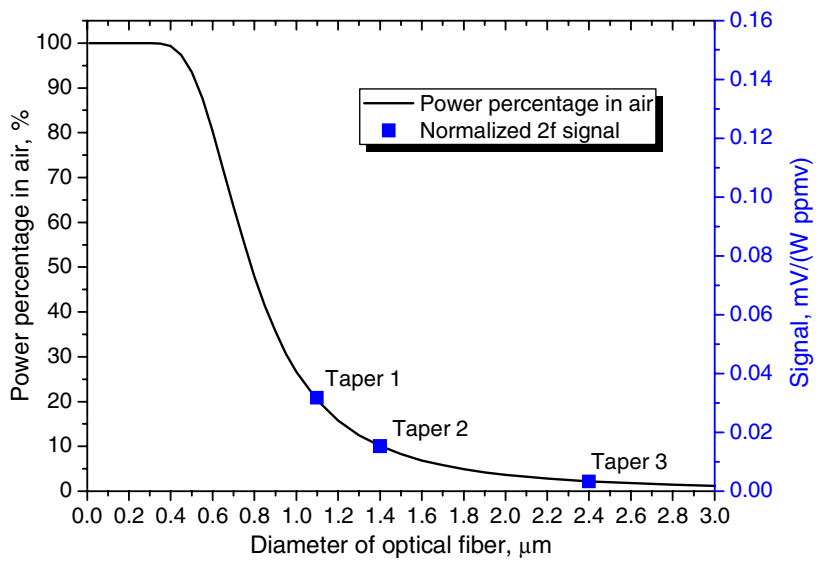

Fig. 4. (Color online) Calculated percentage of light power in the evanescent field for OMNF with varying diameters at $\lambda=$ $1532.8 \mathrm{~nm}$ and measured normalized second-harmonic $(2 f=32.75 \mathrm{kHz})$ signal for three different fiber tapers.

ized by input optical power and gas concentration, are also shown in Fig. 4 as solid squares. As expected, the tendency of the normalized signal agrees well with the theoretical power percentage curve, indicating that the sensitivity may be further improved by reducing the diameter of the fiber taper.

We investigated the dependence of the secondharmonic output on $\mathrm{C}_{2} \mathrm{H}_{2}$ concentration and input optical power. Figures $\underline{5(\mathrm{a})}$ and $5(\mathrm{~b})$ show the results for taper 3 and taper 2, respectively. The output signals have linear relationships with both the gas concentration and the input optical power, which agree well with theoretical predictions in [8]. For different input power levels, the standard deviations of the system noises were calculated and the result for taper 2 is shown in Fig. 5(b). The noise level was found independent of the input optical power and is believed to be mainly due to the amplified thermal

Table 1. Parameters of Three Different OMNFs and Their Performance in QE-EPAS Experiment

\begin{tabular}{lcccccc}
\hline Taper & $\begin{array}{c}\text { Diameter } \\
{[\mu \mathrm{m}]}\end{array}$ & $\begin{array}{c}\text { Power percentage } \\
\text { in Air [\%] }\end{array}$ & $\begin{array}{c}\text { Input Optical } \\
\text { Power [mW] }\end{array}$ & $\begin{array}{c}\text { Maximum } 2 f \\
\text { Signal }[\mathrm{mV}]\end{array}$ & $\begin{array}{c}\mathrm{C}_{2} \mathrm{H}_{2} \\
\text { Concentration }\end{array}$ & $\begin{array}{c}\text { Normalized Maximum } 2 f \\
\text { Signal }[\mathrm{mV} /(\mathrm{W} \text { ppmv)] }\end{array}$ \\
\hline 1 & 1.1 & 20.25 & 9.75 & 4.8822 & $1.58 \%$ & 0.0317 \\
2 & 1.4 & 10.05 & 9.75 & 6.1854 & $4.14 \%$ & 0.0153 \\
3 & 2.4 & 2.18 & 8.37 & 1.0826 & $4 \%$ & 0.0032 \\
\hline
\end{tabular}

${ }^{\mathrm{a} C a l c u l a t e d ~ r e s u l t s ~ a t ~} \lambda=1532.8 \mathrm{~nm}$. 

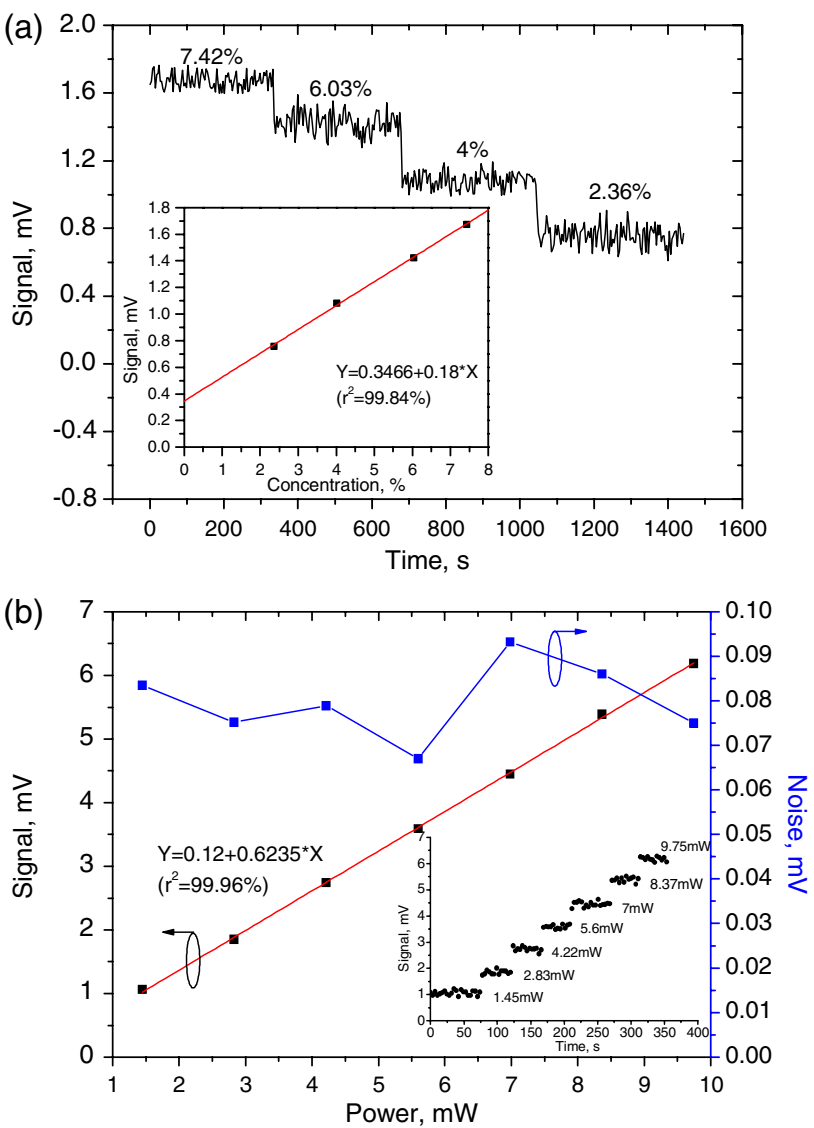

Fig. 5. (Color online) Dependence of second-harmonic output on (a) gas concentration for taper 3 for an input power of $8.37 \mathrm{~mW}$ and (b) input optical power for taper 2 for a gas concentration of $4.14 \%$.

noise of the QTF at a $30 \mathrm{~ms}$ integration time [16]. Based on a measured signal-to-noise ratio of 67.3 for taper 1 with $9.75 \mathrm{~mW}$ input optical power, a normalized noise equivalent absorption (NNEA) coefficient $(1 \sigma)$ of $1.96 \times$ $10^{-6} \mathrm{~cm}^{-1} \cdot \mathrm{W} / \mathrm{Hz}^{1 / 2}$ is estimated.

In conclusion, EPAS with tapered OMNFs was demonstrated for the first time to the best of our knowledge. With a fiber taper of $1.1 \mu \mathrm{m}$ waist diameter and a QTF, we obtain a normalized output signal of $0.0317 \mathrm{mV} /(\mathrm{W}$ ppmv) and an NNEA coefficient of

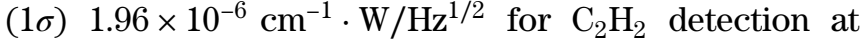

atmospheric pressure. By using a thinner OMNF, a proper acoustic resonator, or detector with better sensitivity such as microcantilever-based interferometric detection [17], the sensitivity would be further improved by 2 to 3 orders of magnitude. Compared with conventional PAS, the all-fiber EPAS has the advantages of easier optical alignment, lower insertion loss, and potential for multipoint multiplexed sensing along a fiber.

We acknowledge the support of the Hong Kong SAR government through a GRF grant PolyU5177/10E and the Hong Kong Polytechnic University through a studentship.

\section{References}

1. G. Whitenett, G. Stewart, K. Atherton, B. Culshaw, and W. Johnstone, J. Opt. A 5, S140 (2003).

2. G. Stewart, W. Jin, and B. Culshaw, Sensors Actuators B 38, 42 (1997).

3. H. Tai, H. Tanaka, and T. Yoshino, Opt. Lett. 12, 437 (1987).

4. Y. L. Hoo, W. Jin, C. Shi, H. L. Ho, D. N. Wang, and S. C. Ruan, Appl. Opt. 42, 3509 (2003).

5. Y. L. Hoo, W. Jin, H. L. Ho, J. Ju, and D. N. Wang, Sensors Actuators B 105, 183 (2005).

6. A. Elia, P. M. Lugarà, C. D. Franco, and V. Spagnolo, Sensors 9, 9616 (2009).

7. A. A. Kosterev, Y. A. Bakhirkin, R. F. Curl, and F. K. Tittel, Opt. Lett. 27, 1902 (2002).

8. A. A. Kosterev, F. K. Tittel, D. V. Serebryakov, A. L. Malinovsky, and I. V. Morozov, Rev. Sci. Instrum. 76, 043105 (2005).

9. S. Schilt, A. A. Kosterev, and F. K. Tittel, Appl. Phys. B 95, 813 (2009).

10. A. Kosterev and F. K. Tittel, Appl. Opt. 43, 6213 (2004).

11. L. Dong, A. A. Kosterev, D. Thomazy, and F. K. Tittel, Appl. Phys. B 100, 627 (2010).

12. K. Liu, X. Guo, H. Yi, W. Chen, W. Zhang, and X. Gao, Opt. Lett. 34, 1594 (2009).

13. L. M. Tong, R. R. Gattass, J. B. Ashcom, S. L. He, J. Y. Lou, M. Y. Shen, I. Maxwell, and E. Mazur, Nature 426, 816 (2003).

14. L. Tong, J. Lou, Z. Ye, G. T. Svacha, and E. Mazur, Nanotechnology 16, 1445 (2005).

15. H. Xuan, W. Jin, and S. Liu, Opt. Lett. 35, 85 (2010).

16. R. D. Grober, J. Acimovic, J. Schuck, D. Hessman, P. J. Kindlemann, J. Hespanha, A. S. Morse, K. Karrai, I. Tiemann, and S. Manus, Rev. Sci. Instrum. 71, 2776 (2000).

17. T. Laurila, H. Cattaneo, V. Koskinen, J. Kauppinen, and R. Hernberg, Opt. Express 13, 2453 (2005). 